BARI-TH 384/00

\title{
Influence of the magnetic field on the fermion scattering off bubble and kink walls
}

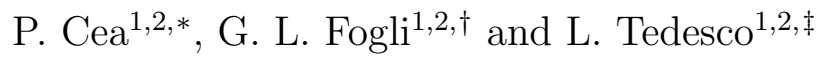 \\ ${ }^{1}$ Dipartimento di Fisica, Università di Bari, I-70126 Bari, Italy \\ ${ }^{2}$ INFN - Sezione di Bari, I-70126 Bari, Italy
}

May, 2000

\begin{abstract}
We investigate the scattering of fermions off domain walls at the electroweak phase transition in presence of a magnetic field. We consider both the bubble wall and the kink domain wall. We derive and solve the Dirac equation for fermions with momentum perpendicular to the walls, and compute the transmission and reflection coefficients. In the case of kink domain wall, we briefly discuss the zero mode solutions localized on the wall. The possibile role of the magnetic field for the electroweak baryogenesis is also discussed.
\end{abstract}

\footnotetext{
*Electronic address: Cea@bari.infn.it

${ }^{\dagger}$ Electronic address: Fogli@bari.infn.it

$\ddagger$ Electronic address: Tedesco@bari.infn.it
} 


\section{INTRODUCTION}

Recently, a considerable amount of theoretical work, based on the Standard Model of electroweak interactions and its extensions, has been done on the hypothesis that the production of the observed baryon asymmetry may be generated at the primordial electroweak phase transition [1]. Indeed, it has been recognized that the baryon number is violated in the Standard Model through the axial anomaly [2]. Thus, it widely believed that the baryon asymmetry may originate in the Standard Model if the electroweak phase transition is of first or weakly first order.

In general, the problem of determining the nature of the electroweak phase transition requires a non perturbative approach. As a matter of fact, in lattice simulations it turns out that the electroweak phase transition is first order for Higgs masses less than $72.1 \pm 1.4 \mathrm{GeV}$, while for larger Higgs masses only a rapid crossover is expected [3]. Thus, if we take into account the lower bound recently established by the LEP Collaborations [⿴囗⿴囗十

$$
M_{H}>95.2 \mathrm{GeV} \text { at } 95 \% \text { C.L. , }
$$

then, we are led to conclude that in the Standard Model there is no first order phase transition. However, a first order phase transition can be obtained with an extension of the Higgs sector of the Standard Model [5], or in the minimal supersymmetric Standard Model [6]. This leaves open the door for a first order phase transition.

On the other hand, even in a perfectly homogeneous continuous phase transition defects will form, if the transition proceeds faster than the order parameter of the broken symmetry phase is able to relax [7]. In such a non equilibrium transition, the new low temperature phase starts to form, due to quantum fluctuations, simultaneously and independently in many parts of the system. Subsequently, these regions grow to form the new broken phase. When different causally disconnected regions meet, the order parameter does not necessarily match and a domain structure is formed. Thus, in the Standard Model the neutral Higgs field is expected to become organised into domains, in each of which the field has a constant sign. As a consequence, the defects are domain walls across which the field flips from one minimum to the other.

The dynamics of the domain walls is governed by the surface tension $\sigma$. In particular, it was pointed out by Zel'dovich, Kobazarev and Okun [8] that the gravitational effects of just one such wall stretched across the universe would introduce a large anisotropy into the relic blackbody radiation. For this reason, the existence of such walls was ruled out. However, it has been suggested recently [9], that the effective surface tension of the domain walls can be made vanishingly small, due to a peculiar magnetic condensation induced by the fermion zero modes localized on the wall. As a consequence, the domain wall acquires a non zero magnetic field perpendicular to the wall and becomes invisible as for as gravitational effects are concerned. In particular, such ferromagnetic walls have been proposed as possible sources of the primordial cosmological magnetic field [9]. On the other hand, even for the bubble walls it has been suggested [10] that strong magnetic fields may be produced as a consequence of non vanishing spatial gradients of the classical value of the Higgs field.

One of the ingredients which enters in the dynamical generation of the baryon asymmetry at the electroweak transition is the estimate of the transmission and reflection coefficients for the fermion scattering off the walls. In other words, it is interesting to see how the magnetic field localized at the wall modifies these coefficients. Aim of this paper is to 
evaluate the reflection and transmission coefficients for the scattering of Dirac fermions off electroweak walls in presence of a magnetic field. We expect that, in general, the magnetic penetration length is much greater than the wall thickness. This means that fermions which scatter on the wall feel an almost constant magnetic field over a spatial region much greater than the wall thickness. So that we can assume that the magnetic field is constant. Moreover, limit our interest to the case of consider magnetic fields perpendicular to the wall and assume the momentum of Dirac fermions asymptotically perpendicular to the wall surface. This corresponds to neglect the motion parallel to the wall surface. As we shall see, this approximation allows to avoid inessential technicalities. The full calculation will be presented in a future work.

The plan of the paper is as follow. In Section 2 we discuss the case of bubble wall. We solve the Dirac equation in presence of a planar bubble wall with a constant magnetic field perpendicular to the wall. We evaluate the reflection and transmission coefficients and compare them with the known results without the magnetic field. Section 3 is devoted to the case of planar domain walls. In this case we discuss the solutions localized on the wall. Finally, some concluding remarks are presented in Section 4.

\section{BUBBLE WALL}

In a first order phase transition the conversion from one phase to another occurs through nucleation of the true phase in the false phase. If the electroweak phase transition is of first order [11], the bubble of the true phase expand and eventually fills the entire volume. At the bubble wall the matter is out of equilibrium and it is here that the baryon asymmetry might be produced. To understand the spontaneous generation of the baryon asymmetry we need to investigate the effects of the bubble wall on the propagation of fermions. Following Ref. [12], we simplify the problem by assuming the bubble, which is at rest, as a planar interface separating the symmetric phase (outside the bubble) from the broken phase (inside the bubble). Fermions passing through the bubble acquire mass, generated by the Yukawa coupling, which is proportional to vacuum expectation value of the Higgs field.

Assuming the bubble wall thickness $\Delta=1$, in our approximation the wall profile reads:

$$
\varphi\left(x_{3}\right)=\frac{v}{2}\left[1+\tanh \left(x_{3}\right)\right]=v g\left(x_{3}\right),
$$

where $x_{3}$ is the direction normal to the wall surface, and $v$ is the vacuum expectation value of the Higgs field.

We are interested in the Dirac equation in the bubble wall background and in presence of the electromagnetic field $A_{\mu}(x)$ :

$$
\left[i \gamma^{\mu} \partial_{\mu}-e \gamma^{\mu} A_{\mu}-g_{Y} \varphi\left(x_{3}\right)\right] \Psi=0
$$

where $e$ and $g_{Y}$ are the electric and Yukawa coupling respectively. To solve Eq. (2.2) we assume:

$$
\Psi=\left[i \gamma^{\mu} \partial_{\mu}-e \gamma^{\mu} A_{\mu}+g_{Y} \varphi\left(x_{3}\right)\right] \Phi .
$$

Inserting into Eq. (2.2) we readily obtain:

$$
\left[-\partial^{2}-2 i e A_{\mu} \partial^{\mu}+e^{2} A_{\mu} A^{\mu}-\xi^{2} g^{2}\left(x_{3}\right)+i \xi \gamma^{\mu} \partial_{\mu} g\left(x_{3}\right)-\frac{1}{2} \sigma^{\mu \nu} F_{\mu \nu}\right] \Phi=0,
$$


where $2 \xi$ is the mass which the fermion acquires in the broken phase. In the case of constant magnetic field directed along the third spatial direction we have $F_{12}=B$. It is easy to see that the solutions of Eq. (2.4) factorize as:

$$
\Phi\left(x_{1}, x_{2}, x_{3}, t\right)=\zeta\left(x_{1}, x_{2}\right) \phi\left(x_{3}, t\right)
$$

where $\zeta$ is a scalar function which describes the motion transverse to the bubble wall. In this paper we consider particles with momentum perpendicular to the wall. Then Eq. (2.4) reduces to:

$$
\left(-\partial_{t}^{2}-\partial_{3}^{2}-\xi^{2} g^{2}\left(x_{3}\right)+i \xi \gamma^{3} \partial_{3} g\left(x_{3}\right)-e \sigma^{12} F_{12}\right) \phi\left(x_{3}, t\right)=0 .
$$

Putting $\phi\left(x_{3}, t\right)=e^{\mp i E t} \omega\left(x_{3}\right)$, corresponding to positive and negative energy solutions respectively, we get:

$$
\left(\frac{d^{2}}{d x_{3}^{2}}+i \gamma^{3} \frac{d g}{d x_{3}}-\xi^{2} g^{2}\left(x_{3}\right)+E^{2}+i e B \gamma^{1} \gamma^{2}\right) \omega\left(x_{3}\right)=0
$$

In order to solve Eq. (2.7), we expand $\omega\left(x_{3}\right)$ in terms of the spinors $u_{ \pm}^{s}$ eigenstates of $\gamma^{3}$ :

$$
\omega(z)=\phi_{+}^{1} u_{+}^{1}+\phi_{-}^{1} u_{-}^{1}+\phi_{+}^{2} u_{+}^{2}+\phi_{-}^{2} u_{-}^{2} .
$$

Using the standard representation for the Dirac matrices [13] we find:

$$
\begin{aligned}
& u_{ \pm}^{1}=\left(\begin{array}{c}
1 \\
0 \\
\pm i \\
0
\end{array}\right), \\
& u_{ \pm}^{2}=\left(\begin{array}{c}
0 \\
1 \\
0 \\
\mp i
\end{array}\right)
\end{aligned}
$$

It is straightforward to check that:

$$
\begin{aligned}
\gamma^{3} u_{ \pm}^{1,2} & = \pm i u_{ \pm}^{1,2} \\
\gamma^{0} u_{ \pm}^{1,2} & =+u_{\mp}^{1,2} \\
\gamma^{1} \gamma^{2} u_{ \pm}^{1} & =-i u_{ \pm}^{1} \\
\gamma^{1} \gamma^{2} u_{ \pm}^{2} & =+i u_{ \pm}^{2}
\end{aligned}
$$

Taking into account Eqs. (2.8),(2.9),(2.10), (2.11) we obtain the equations:

$$
\begin{aligned}
& \left(\frac{d^{2}}{d x_{3}^{2}} \mp \xi \frac{d g}{d z}-\xi^{2} g^{2}\left(x_{3}\right)+E^{2}+e B\right) \phi_{ \pm}^{1}=0, \\
& \left(\frac{d^{2}}{d x_{3}^{2}} \mp \xi \frac{d g}{d x_{3}}-\xi^{2} g^{2}\left(x_{3}\right)+E^{2}-e B\right) \phi_{ \pm}^{2}=0 .
\end{aligned}
$$


As, expected the magnetic field introduces an explicit spin dependence in the equations. Note that, in our approximation the spin projection on the third spatial axis coincides with the helicity.

In order to solve the differential equations (2.12), (2.13), following Ref. [12] we introduce the new variable:

$$
y=\frac{1}{1+e^{2 x_{3}}} .
$$

For definiteness, let us consider Eq. (2.12). We have:

$$
\left[\frac{d^{2}}{d y^{2}}+\frac{1-2 y}{y(1-y)} \frac{d}{d y}+\frac{E^{2}+e B \mp 4 \xi y(1-y)-4 \xi^{2}(1-y)^{2}}{4 y^{2}(1-y)^{2}}\right] \phi_{ \pm}^{1}=0
$$

By examining the behaviour of the differential equation near the singular points, we get:

$$
\phi_{ \pm}^{1}\left(x_{3}\right)=y^{\alpha^{1}}(1-y)^{\beta^{1}} \chi_{ \pm}^{1}(y)
$$

where $\chi_{ \pm}^{1}(y)$ is regular near the singularities. A standard calculation gives:

$$
\begin{aligned}
\alpha^{1} & =\frac{i}{2} \sqrt{E^{2}+e B-4 \xi^{2}} \\
\beta^{1} & =\frac{i}{2} \sqrt{E^{2}+e B} .
\end{aligned}
$$

Inserting Eq. (2.16) into Eq. (2.15) one sees that $\chi_{ \pm}^{1}(y)$ satisfies the hypergeometric equation [14] with parameters:

$$
\begin{aligned}
& a_{\mp}^{1}=\alpha^{1}+\beta^{1}+\frac{1}{2}-\left|\xi \mp \frac{1}{2}\right|, \\
& b_{\mp}^{1}=\alpha^{1}+\beta^{1}+\frac{1}{2}+\left|\xi \mp \frac{1}{2}\right|, \\
& c^{1}=2 \alpha^{1}+1 .
\end{aligned}
$$

As well known, the general solution of the hypergeometric equation is a combination of the two independent solutions:

$$
\begin{array}{r}
{ }_{2} F_{1}\left(a_{\mp}, b_{\mp}, c, y\right), \\
y^{1-c}{ }_{2} F_{1}\left(a_{\mp}+1-c, b_{\mp}+1-c, 2-c ; y\right) .
\end{array}
$$

Therefore:

$$
\begin{aligned}
\phi_{ \pm}^{1}= & A_{ \pm}^{1} y^{\alpha^{1}}(1-y)_{2}^{\beta^{1}} F_{1}\left(a_{\mp}^{1}, b_{\mp}^{1}, c^{1}, y\right)+ \\
& +B_{ \pm}^{1} y^{-\alpha^{1}}(1-y)^{\beta^{1}}{ }_{2} F_{1}\left(a_{\mp}^{1}+1-c^{1}, b_{\mp}^{1}+1-c^{1}, 2-c^{1} ; y\right),
\end{aligned}
$$

where $A_{ \pm}^{1}$ and $B_{ \pm}^{1}$ are normalization constants. For simplicity we define:

$$
\begin{aligned}
& \phi_{ \pm}^{\left(-\alpha^{1}\right)}=y^{\alpha^{1}}(1-y)_{2}^{\beta^{1}} F_{1}\left(a_{\mp}^{1}, b_{\mp}^{1}, c^{1} ; y\right) \\
& \phi_{ \pm}^{\left(+\alpha^{1}\right)}=y^{-\alpha^{1}}(1-y)^{\beta^{1}}{ }_{2} F_{1}\left(a_{\mp}^{1}+1-c^{1}, b_{\mp}^{1}+1-c^{1}, 2-c^{1} ; y\right) .
\end{aligned}
$$


The fermionic wave function can be obtained from Eq. (2.3), which in our approximation reduces to:

$$
\Psi^{1}\left(x_{3}, t\right)=\left[-i \gamma^{0} \partial_{0}+i \gamma^{3} \partial_{3}+\xi g\left(x_{3}\right)\right] e^{\mp i E t} \phi_{+}^{1} u_{+}^{1}
$$

A calculation similar to that performed in Ref. [12] gives:

$$
\begin{aligned}
\Psi^{1}\left(x_{3}\right)= & A^{1}\left[\epsilon_{r} \phi_{+}^{1{ }^{\left(-\alpha^{1}\right)}} u_{-}^{1}+2\left(\xi+\alpha^{1}\right) \phi_{-}^{1{ }^{\left(-\alpha^{1}\right)}} u_{+}^{1}\right]+ \\
& B^{1}\left[\epsilon_{r} \phi_{+}^{1{ }^{\left(+\alpha^{1}\right)}} u_{-}^{1}+2\left(\xi-\alpha^{1}\right) \phi_{-}^{1{ }^{\left(+\alpha^{1}\right)}} u_{+}^{1}\right]
\end{aligned}
$$

where $\epsilon_{r}=E$ for $r=1$ and $\epsilon_{r}=-E$ for $r=2$.

We are interested in fermions incident from the symmetric phase region $\left(x_{3} \rightarrow-\infty\right)$, reflected in part by the bubble wall and transmitted in the broken phase region $\left(x_{3} \rightarrow\right.$ $+\infty)$. We need then the asymptotic forms of $\phi_{ \pm}^{\left(-\alpha^{1}\right)}$ and $\phi_{ \pm}^{\left(+\alpha^{1}\right)}$ :

$$
\begin{aligned}
& \lim _{x \rightarrow+\infty} y^{ \pm \alpha^{1}}\left(1-y^{\beta^{1}}\right)=\exp \left(\mp 2 \alpha^{1} x_{3}\right), \\
& \lim _{x \rightarrow-\infty} y^{\alpha^{1}}\left(1-y^{ \pm \beta^{1}}\right)=\exp \left( \pm 2 \beta^{1} x_{3}\right) .
\end{aligned}
$$

After some algebra we obtain the transmitted, incident and reflected wave functions:

$$
\begin{gathered}
\left(\Psi^{1}\right)^{\text {trans }}=A\left[\epsilon_{r} u_{-}^{1}+2\left(\xi-\alpha^{1}\right) u_{+}\right] \exp \left(2 \alpha^{1} x\right) \\
\left(\Psi^{1}\right)^{i n c}=A \frac{\Gamma\left(1-2 \alpha^{1}\right) \Gamma\left(-2 \beta^{1}\right)}{\Gamma\left(-\alpha^{1}-\beta^{1}+\xi\right) \Gamma\left(-\alpha^{1}-\beta^{1}-\xi\right)}\left[\frac{\epsilon_{r} u_{-}^{1}}{-\alpha^{1}-\beta^{1}-\xi}+\frac{2\left(\xi-\alpha^{1}\right) u_{+}^{1}}{-\alpha^{1}-\beta^{1}+\xi}\right] e^{2 \beta^{1} x_{3}}, \\
\left(\Psi^{1}\right)^{r e f}=A \frac{\Gamma\left(1-2 \alpha^{1}\right) \Gamma\left(2 \beta^{1}\right)}{\Gamma\left(-\alpha^{1}+\beta^{1}+\xi\right) \Gamma\left(-\alpha^{1}+\beta^{1}-\xi\right)}\left[\frac{\epsilon_{r} u_{-}^{1}}{-\alpha^{1}+\beta^{1}-\xi}+\frac{2\left(\xi-\alpha^{1}\right) u_{+}^{1}}{-\alpha^{1}+\beta^{1}+\xi}\right] e^{-2 \beta^{1} x_{3} .}
\end{gathered}
$$

The relevant current turns out to be:

$$
j_{V}^{3}=\bar{\psi} \gamma_{3} \psi
$$

Taking into account Eqs. (2.26), (2.27), (2.28), we get:

$$
\begin{gathered}
\left(j_{V}^{3}\right)^{1, i n c}=8 i \epsilon_{r} \beta^{1}|A|^{2}\left|\frac{\Gamma\left(1+2 \alpha^{1}\right) \Gamma\left(2 \beta^{1}\right)}{\Gamma\left(\alpha^{1}+\beta^{1}+\xi\right)+\Gamma\left(\alpha^{1}+\beta^{1}-\xi\right)}\right|^{2} \frac{1}{\left(\alpha^{1}+\beta^{1}+\xi\right)\left(\alpha^{1}+\beta^{1}-\xi\right)}, \\
\left(j_{V}^{3}\right)^{1, \text { trans }}=-8 i \epsilon_{r} \alpha^{1}|A|^{2}
\end{gathered}
$$




$$
\left(j_{V}^{3}\right)^{1, r e f l}=-8 i \epsilon_{r} \beta^{1}|A|^{2}\left|\frac{\Gamma\left(1+2 \alpha^{1}\right) \Gamma\left(2 \beta^{1}\right)}{\Gamma\left(-\alpha^{1}+\beta^{1}+\xi\right) \Gamma\left(-\alpha^{1}+\beta^{1}-\xi\right)}\right|^{2} \frac{1}{\left(\alpha^{1}-\beta^{1}-\xi\right)\left(\alpha^{1}-\beta^{1}+\xi\right)}
$$

By means of the identity

$$
\Gamma(z) \Gamma(-z)=\frac{-\pi}{z \sin (\pi z)}
$$

we obtain the following reflection and transmission coefficients:

$$
\begin{aligned}
& R^{1}=\frac{\sin \left[\pi\left(\alpha^{1}-\beta^{1}+\xi\right)\right] \sin \left[\pi\left(-\alpha^{1}+\beta^{1}+\xi\right)\right]}{\sin \left[\pi\left(-\alpha^{1}-\beta^{1}+\xi\right)\right] \sin \left[\pi\left(+\alpha^{1}+\beta^{1}+\xi\right)\right]} \\
& T^{1}=\frac{\sin \left(2 \pi \alpha^{1}\right) \sin \left(2 \pi \beta^{1}\right)}{\sin \left[\pi\left(-\alpha^{1}-\beta^{1}+\xi\right) \sin \left[\pi\left(+\alpha^{1}+\beta^{1}+\xi\right)\right]\right.},
\end{aligned}
$$

where the superscript 1 refers to the spin projection.

Note that the magnetic field influences the coefficients by means the parameters $\alpha^{1}, \beta^{1}$. It is easily checked that $R^{2}$ and $T^{2}$ are given by the same Eqs. (2.34) and (2.35) with $\alpha^{1}$ and $\beta^{1}$ replaced by:

$$
\begin{aligned}
\alpha^{2} & =\frac{i}{2} \sqrt{E^{2}-e B-4 \xi^{2}} \\
\beta^{2} & =\frac{i}{2} \sqrt{E^{2}-e B}
\end{aligned}
$$

which, as expected, correspond to $B \longrightarrow-B$. One can verify that $R+T=1$ and that for $B=0 R^{1,2}$ and $T^{1,2}$ agree with the results of Ref. [12].

In Figure 1 we compare the behaviour of $R^{1}$ and $R^{2}$ as a function of the scaled energy $E / 2 \xi$ with the zero magnetic field reflection coefficient. We see that the magnetic field is able to produce an asymmetry in the spin distribution, but there is no particle-antiparticle asymmetry, which is relevant in the electroweak baryogenesis. In particular, there is total reflection for fermions with antiparallel spin and energies $E^{2}-4 \xi^{2} \leq e B$.

In Figure 2 we report the reflection coefficients at a given energy as a function of $e B$. We see that by increasing the magnetic field the reflection coefficient decreases for fermions with parallel spin, and increases for antiparallel spin. Thus the magnetic field enhances the spin asymmetry in the scattering off the bubble wall.

\section{KINK DOMAIN WALL}

In this Section we consider the domain walls which are thought to be formed in a continuous phase transition by the Kibble mechanism [7]. Indeed, if the scalar field develops a non vanishing vacuum expectation value $\langle\phi\rangle= \pm v$, then in general one may assume that there are regions with $\langle\phi\rangle=+v$ and $\langle\phi\rangle=-v$. It is easy to see that the classical equation of motion of the scalar field admits the solution describing the transition layer between 
two adjacent regions with different values of $\langle\phi\rangle$ (assuming the domain wall thickness $\Delta=1)$ :

$$
\varphi\left(x_{3}\right)=v \tanh \left(x_{3}\right)=v g\left(x_{3}\right) .
$$

The solution (3.1) is known as kink. In the same approximation of the previous Section we must solve the Dirac equation (2.7) with $g\left(x_{3}\right)$ given by Eq. (3.1), $\xi$ being the fermion mass in the broken phase. Again we write the spinor $\phi\left(x_{3}\right)$ in the basis (2.9) and get four different equations. For definiteness we focus on the equation for $\phi\left(x_{3}\right)=\phi_{+}^{1}\left(x_{3}\right) u_{+}^{1}$. We obtain:

$$
\left(\frac{d^{2}}{d x_{3}^{2}}-\xi \frac{d g}{d x_{3}}-\xi^{2} g^{2}\left(x_{3}\right)+E^{2}+e B\right) \phi_{+}^{1}=0 .
$$

Following the approach of the previous Section, we find:

$$
\phi_{+}^{1}\left(x_{3}\right)=y^{\alpha^{1}}(1-y)^{\alpha^{1}} \chi_{+}^{1}(y)
$$

where $\chi_{+}^{1}(y)$ is regular near the singularities, $y$ is defined in Eq. (2.14) and:

$$
\alpha^{1}=\frac{i}{2} \sqrt{E^{2}+e B-\xi^{2}}
$$

Again we find that $\chi_{+}^{1}(y)$ satisfies a hypergeometric equation with parameters:

$$
\begin{aligned}
& a=2 \alpha^{1}+\frac{1}{2}-\left|\xi-\frac{1}{2}\right|, \\
& b=2 \alpha^{1}+\frac{1}{2}+\left|\xi-\frac{1}{2}\right|, \\
& c=2 \alpha^{1}+1 .
\end{aligned}
$$

After some calculations we obtain the general solution of the Dirac equation. We have:

$$
\begin{aligned}
\Psi^{1}\left(x_{3}\right)= & A^{1} y^{\alpha^{1}}(1-y)^{\alpha^{1}}\left[E_{2} F_{1}\left(2 \alpha^{1}+\xi, 2 \alpha^{1}-\xi+1,2 \alpha^{1}+1 ; y\right) u_{-}^{1}+\right. \\
& \left.+\left(\xi+2 \alpha^{1}\right){ }_{2} F_{1}\left(2 \alpha^{1}-\xi, 2 \alpha^{1}+\xi+1,2 \alpha^{1}+1 ; y\right) u_{+}^{1}\right]+ \\
& +B^{1} y^{-\alpha^{1}}(1-y)^{\alpha^{1}}\left[E_{2} F_{1}\left(\xi, 1-\xi, 1-2 \alpha^{1} ; y\right) u_{-}^{1}+\right. \\
& \left.+\left(\xi-2 \alpha^{1}\right){ }_{2} F_{1}\left(-\xi, 1+\xi, 1-2 \alpha^{1} ; y\right) u_{+}^{1}\right] .
\end{aligned}
$$

Note that in the present case both the regions $x_{3} \rightarrow \pm \infty$ correspond to the broken phase. The case of a fermion incident from $x_{3} \rightarrow-\infty$ is given by Eq. (3.6) with $A^{1}=0$. Using the asymptotic expansion of the hypergeometric functions [14, we obtain the incident, reflected and transmitted wave functions:

$$
\begin{gathered}
\left(\Psi^{1}\right)^{i n c}=B^{1} \frac{\Gamma\left(1-2 \alpha^{1}\right) \Gamma\left(-2 \alpha^{1}\right)}{\Gamma\left(-2 \alpha^{1}+\xi\right) \Gamma\left(-2 \alpha^{1}-\xi\right)}\left[\frac{E u_{-}^{1}}{-2 \alpha^{1}-\xi}+u_{+}^{1}\right] e^{2 \alpha^{1} x_{3}}, \\
\left(\Psi^{1}\right)^{r e f}=B^{1} \frac{\Gamma\left(1-2 \alpha^{1}\right) \Gamma\left(2 \alpha^{1}\right)}{\Gamma(\xi) \Gamma(-\xi)}\left[\frac{E u_{-}^{1}}{-\xi}+\frac{\left.\xi-2 \alpha^{1}\right) u_{+}^{1}}{\xi}\right] e^{-2 \alpha^{1} x_{3}},
\end{gathered}
$$




$$
\left(\Psi^{1}\right)^{\text {trans }}=B^{1}\left[E u_{-}^{1}+\left(\xi-2 \alpha^{1}\right) u_{+}^{1}\right] e^{2 \alpha^{1} x_{3}} .
$$

Finally, the reflection and transmission coefficients are given by:

$$
\begin{aligned}
R^{1} & =\frac{\sin ^{2}(\pi \xi)}{\sin \left[\pi\left(-2 \alpha^{1}+\xi\right)\right] \sin \left[\pi\left(+2 \alpha^{1}+\xi\right)\right]}, \\
T^{1} & =\frac{\sin \left(2 \pi \alpha^{1}\right) \sin \left(-2 \pi \alpha^{1}\right)}{\sin \left[\pi\left(-2 \alpha^{1}+\xi\right)\right] \sin \left[\pi\left(+2 \alpha^{1}+\xi\right)\right]} .
\end{aligned}
$$

$R^{2}$ and $T^{2}$ are easily obtained from the previous equations by $B \rightarrow-B$. The dependence of the reflection and transmission coefficients on the magnetic field is quite similar to the bubble wall case (see Fig. 3).

Let us conclude this Section by discussing the fermion states localized on the wall. Indeed, it is known that the differential equation:

$$
\left(\frac{d^{2}}{d x_{3}^{2}}+i \gamma^{3} \frac{d g}{d x_{3}}-\xi^{2} g^{2}\left(x_{3}\right)+E^{2}+i e B \gamma^{1} \gamma^{2}\right) \omega\left(x_{3}\right)=0
$$

admits zero energy solutions localized on the wall in absence of the magnetic field [15]. For the scattering problem we are discussing, these solutions are not relevant. However, in presence of a non zero magnetic field it is easy to see that there are localized states if:

$$
E^{2} \pm e B=0
$$

Thus, we see that there is a localized state:

$$
\omega\left(x_{3}\right)=e^{-\kappa\left(x_{3}\right)} u_{-}^{2}, \quad \frac{d \kappa}{d x_{3}}=\xi g\left(x_{3}\right)
$$

when $E^{2}=e B$. In general, if we take into account the motion parallel to the wall, it can be shown that there are localized states for $E^{2}=2 n e B, n=1,2, \ldots$.

It is worthwhile to stress that the localized states, which are a peculiar characteristic of the domain walls, may play an important role in the dynamics of the walls.

\section{CONCLUSIONS}

In this paper we have investigated the effects of a constant magnetic field on the scattering of fermions on planar walls. We have discussed both the bubble wall, which is relevant for a first order electroweak phase transition, and the kink domain wall. In particular, we solved the Dirac equation for fermions with momentum perpendicular to the walls, and computed the transmission and reflection coefficients. As expected, we find that the constant magnetic field induces a spin asymmetry in the fermion reflections and transmissions. The results obtained are interesting, but do not allow to produce directly asymmetries in some local charges which is a prerequisite for the idea of electroweak baryogenesis [1]. However, before reaching any conclusions, one should take into account 
that the magnetic field distorts the fermion thermal equilibrium distribution, so that it could in principle induce an asymmetry between fermion and antifermion distributions. For instance, if the magnetic field modifies the chemical potential, then the fermion and antifermion distributions are expected to be different. Such an analysis, however, requires the study of the quantum Boltzmann transport equation [16].

Let us conclude this paper by briefly discussing the role of the domain walls in the electroweak baryogenesis. Indeed, the baryon number dynamical generation induced by bubble walls has been extensively discussed in the literature. Coversely, the possible role of kink domain walls has never discussed before, due to the fact that the existence of such walls was ruled out by the Zel'dovich, Kobazarev and Okun argument [8]. As we argued before, ferromagnetic domain walls with vanishing effective surface tension are an open possibility [9]. As a consequence, it is worthwhile to address ourself on possible mechanisms to generate baryon number by domain walls.

As well known, the baryon number violation in the electroweak theory takes place through saddle-point configurations called sphalerons. In general, the sphaleron mechanism is not exponentially suppressed in regions where the vacuum expectation value of the Higgs field is small. Thus, in the case of domain walls, the sphaleron mediated baryon number generation can be active only in the small transition layer of the wall. Apparently this fact seems to rule out any relevance of the domain walls in the electroweak baryogenesis. However, in Section 3 we find that the kink domains in a constant magnetic field display fermion states localized on the wall. For these trapped fermions the sphaleron could efficiently converts any local charge asymmetry into a baryon number asymmetry, and these processes could be active for a long time and spread over a large region of the primordial universe. If this is the case, we see that the actual value of the baryon number asymmetry could be accounted for even with the tiny amount of Standard Model CP violation. 


\section{References}

[1] For a recent review, see: M. Trodden, Rev. Mod. Phys. 71 (1999) 1463; A. Riotto, Theories of Baryongenesis, lectures delivered at the Summer School in High Energy Physics and Cosmology, Miramare-Trieste, July 1998, hep-ph/9807454.

[2] G. 't Hooft, Phys. Rev. Lett. 37 (1976) 8; V. Kuzmin,V. Rubakov, and M. Shaposhnikov, Phys. Lett. B155 (1985) 36.

[3] For a recent review, see: Z. Fodor, Nucl. Phys. (Proc. Suppl.) 83-84 (2000) 121.

[4] ALEPH, DELPHI, L3, OPAL and LEP Working Group for Higgs Boson Searches, Technical Report No. ALEPH 99-081 CONF 99-052, International Europhysics Conference on High Energy Physics, Tampere, Finland.

[5] M. E. Shaposhnikov, JETP Lett. 44 (1986) 465; Nucl. Phys. B287 (1987) 757; Nucl. Phys. B299 (1988) 797; L. McLerran, Phys. Rev. Lett. 62 (1989) 1075; N. Turok and J. Zadrozny, Nucl. Phys. B 358 (1991)471; Nucl. Phys. B 369 (1992) 729; M. Joyce, T. Prokopec, and N. Turok, Phys. Rev. D58 (1995) 1234 ; J. M. Cline and A. P. Vischer, Phys. Rev. D 54 (1996) 2451.

[6] D. Comelli, M. Pietroni, and A. Riotto, Nucl. Phys. B412 (1994) 441.

[7] T. W. B. Kibble, J. Phys. A9 (1976) 1387; Phys. Rep. 67 (1980) 183.

[8] Ya. B. Zeld'dovich, I. Ya. Kobzarev, and L. B. Okun, JETP. 40 (1974) 1.

[9] P. Cea and L. Tedesco, Phys. Lett. B450 (1999) 61.

[10] T. Vachaspati, Phys. Lett. B265 (1991) 258. See also: D. Grasso and A. Riotto, Phys. Lett. B418 (1998) 258.

[11] D. A. Kirzhnitz, JETP Lett. 15 (1972) 529; D. A. Kirzhnitz and A. D. Linde, Phys. Lett. 72B (1972) 471.

[12] A. Ayala, J. Jalilian-Marian, L. McLerran and A. P. Vischer, Phys. Rev. D49 (1994) 5559 .

[13] J. D. Bjorken and S. Drell, Relativistic Quantum Fields, McGraw Hill, New York (1962).

[14] I. S. Gradshteyn and I. M. Ryzshik, Table of Integrals, Series and Products, Academic Press, New York (1963).

[15] R. Jackiw and C. Rebbi, Phys. Rev. D13 (1976) 3398; A. T. Niemi and G. W. Semenoff, Phys. Rep. C 135 (1986) 100.

[16] L. P. Kadanoff and A. Baym, Quantum Statistical Mechanics, W. A. Benjamin (1962); E. M. Lifshitz and L. P. Pitaevskii, Physical Kinetics, Pergamon Press (1979). 


\section{List of Figures}

$1 \quad$ Bubble wall reflection coefficients in terms of the scaled energy $E / 2 \xi$. The dashed and dotted lines correspond to $e B=0.1$ and parallel and antiparallel spin respectively, and are to be compared with the case $B=0$ (solid

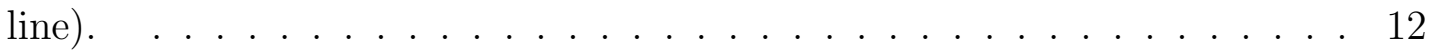

$2 \quad$ Bubble wall reflection coefficients for parallel ( solid line) and antiparallel

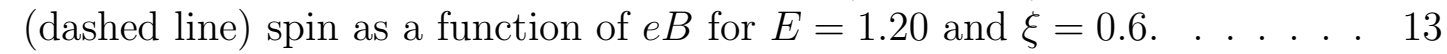

3 Reflection coefficients in the case of the domain wall. Symbols as in Fig. 1.14 


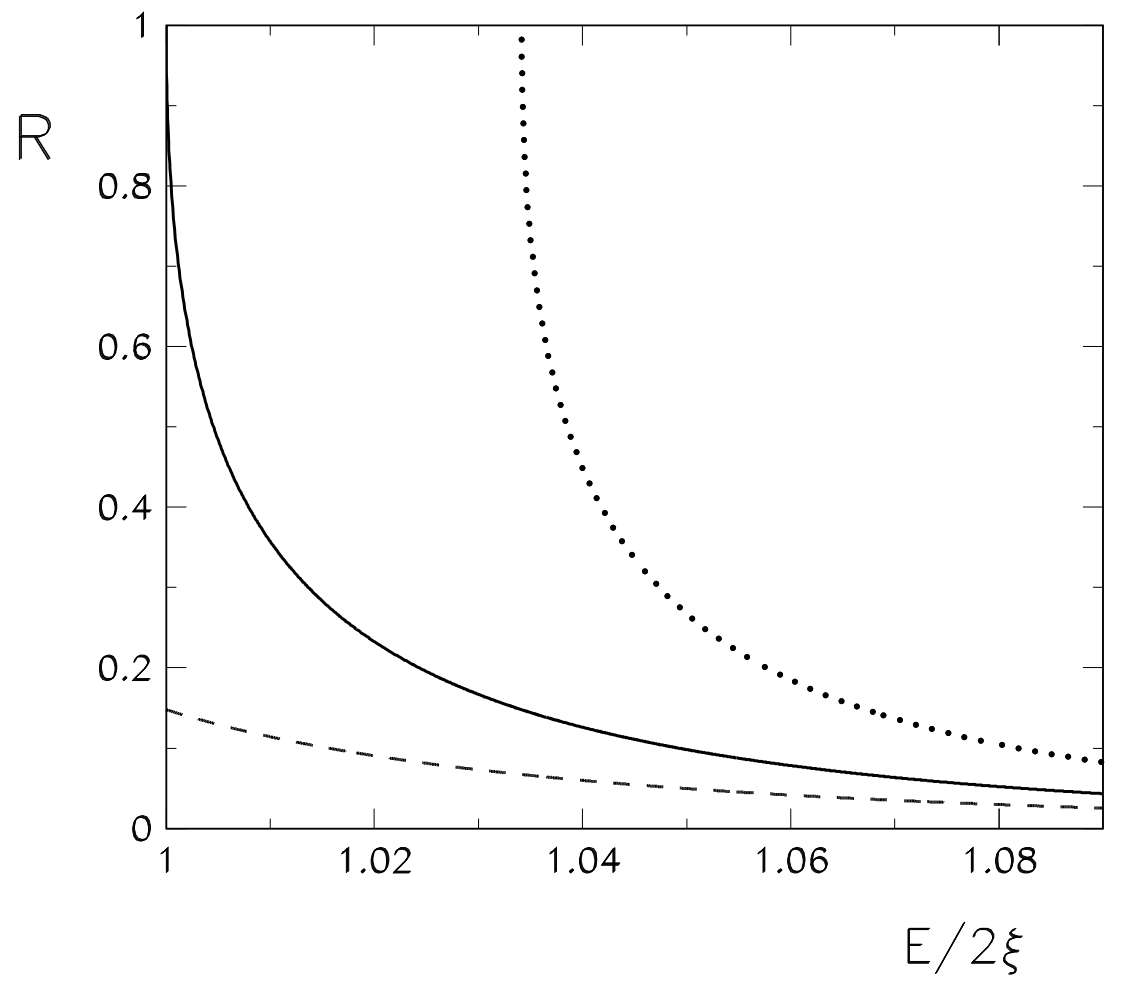

Figure 1: Bubble wall reflection coefficients in terms of the scaled energy $E / 2 \xi$. The dashed and dotted lines correspond to $e B=0.1$ and parallel and antiparallel spin respectively, and are to be compared with the case $B=0$ (solid line). 


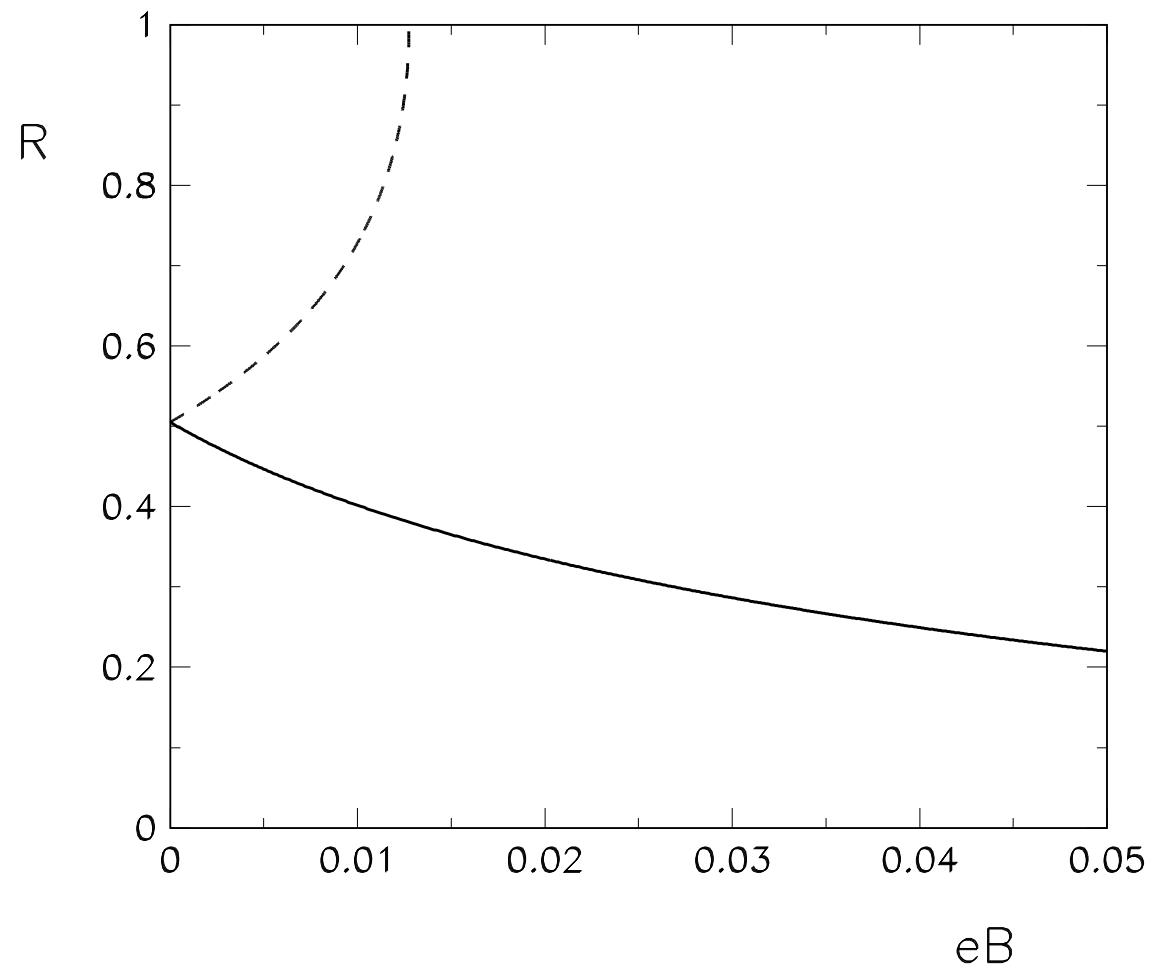

Figure 2: Bubble wall reflection coefficients for parallel ( solid line) and antiparallel (dashed line) spin as a function of $e B$ for $E=1.20$ and $\xi=0.6$. 


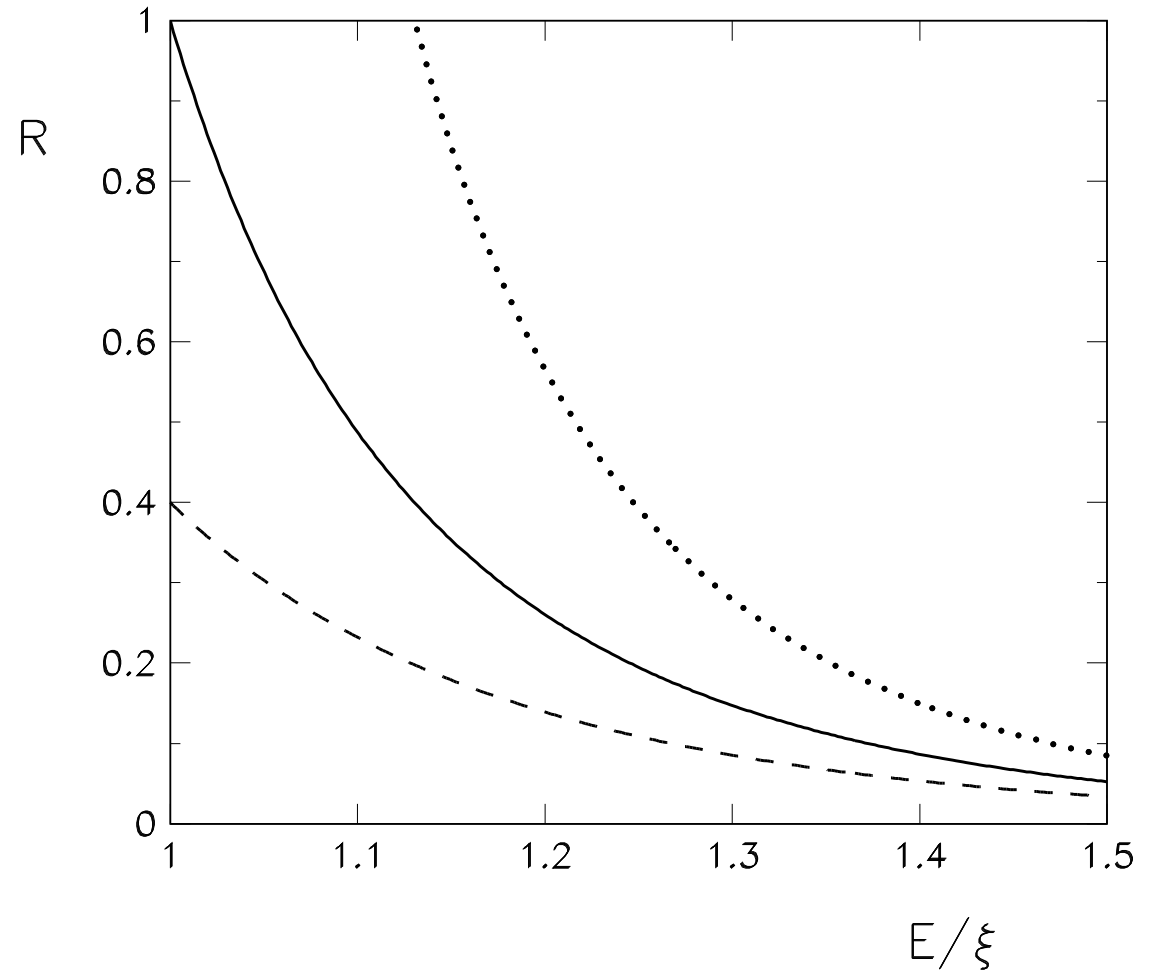

Figure 3: Reflection coefficients in the case of the domain wall. Symbols as in Fig. 1. 